\title{
Adjuvant Radiotherapy for Breast Cancer: More than Meets the Eye
}

\author{
David Krug \\ Department of Radiation Oncology, University Hospital Schleswig-Holstein, Kiel, Germany
}

Radiotherapy has been used in the adjuvant treatment of breast cancer patients for several decades. Level 1 evidence has demonstrated improvements in locoregional control, breast cancer-specific survival, and overall survival in the setting of whole-breast radiotherapy after breast-conserving surgery, regional nodal irradiation, and postmastectomy radiotherapy [1-3]. However, times are changing. Radiotherapy is no longer a one-size-fits-all approach [4]. Numerous clinical trials have led to a significant increase in the level of complexity in the management of breast cancer patients $[5,6]$. Biological risk stratification, systemic therapy, and surgical radicality have changed dramatically, as have the available options regarding individualization of radiotherapy in terms of fractionation and target volume.

In this special issue of Breast Care, the spotlight is on several important aspects of radiotherapy in this modern era.

The indication for adjuvant radiotherapy is mainly based on clinicopathologic factors such as patient age, tumor size, and lymph node involvement as well as hormone and HER2 receptor status. However, gene expression assays are commercially available and they are increasingly being used to determine the indication for adjuvant chemotherapy. Thus, patients frequently present for discussion of adjuvant radiotherapy with results from these assays. One of the articles accompanying this editorial is a review of the literature regarding the question of whether these readily available results may be used to determine the need for adjuvant radiotherapy [7].
Accelerated partial breast irradiation (APBI) is an exciting concept for patients with low-risk early-stage breast cancer. Targeting only the tumor bed and a clinical safety margin around it instead of the whole breast allows for the use of higher doses per fraction, a significant reduction of the overall treatment time, and, potentially, a decrease in the risk of side effects from adjuvant radiotherapy. However, randomized clinical trials studying APBI have used various techniques, fractionations, and study designs which demand a thorough examination. Forster et al. [8] summarize the current evidence on this topic, including recently published data, and present novel developments in terms of ongoing trials and technical improvements such as magnetic resonance-guided radiotherapy.

Regional nodal irradiation has seen a renaissance for early-stage breast cancer after publication of several trials demonstrating improved oncologic outcome with regional nodal irradiation including the internal mammary lymph nodes [9-11]. However, the techniques applied for radiotherapy delivery and the treated target volumes in these trials were outdated $[12,13]$. Duma [14] summarizes the available clinical evidence as well as new and provocative data with implications for target volume definition.

Last but not least, Matuschek et al. [15] present the concept of neoadjuvant radiotherapy for breast cancer. Neoadjuvant radio(chemo)therapy is a concept well known to radiation oncologists and it is considered standard of care for many oncological diseases such as esoph- 
ageal cancer, rectal cancer, and soft-tissue sarcoma. There are some historical trials using neoadjuvant radiotherapy in breast cancer, but it is not routinely used nowadays [16]. The authors provide an overview of the literature as a rationale for the planned phase $3 \mathrm{NeoRad}$ trial.

In summary, these are exciting times for radiation oncologists. However, detailed knowledge of the available evidence is crucial for interdisciplinary discussions and for providing our patients with optimal guidance during the decision-making process.

\section{Disclosure Statement}

D. Krug has received personal fees from Merck Sharp \& Dohme outside of the submitted work.

\section{Funding Sources}

No funding was provided for this work.

\section{References}

1 Darby S, McGale P, Correa C, Taylor C, Arriagada $\mathrm{R}$, Clarke $\mathrm{M}$, et al.; Early Breast Cancer Trialists' Collaborative Group (EBCTCG). Effect of radiotherapy after breast-conserving surgery on 10-year recurrence and 15-year breast cancer death: meta-analysis of individual patient data for 10,801 women in 17 randomised trials. Lancet. 2011 Nov;378(9804): 1707-16.

2 McGale P, Taylor C, Correa C, Cutter D, Duane F, Ewertz M, et al.; Early Breast Cancer Trialists' Collaborative Group. Effect of radiotherapy after mastectomy and axillary surgery on 10-year recurrence and 20-year breast cancer mortality: meta-analysis of individual patient data for 8,135 women in 22 randomised trials. Lancet. 2014 Jun;383(9935): 2127-35.

3 Early Breast Cancer Trialists' Collaborative Group; Dodwell D, Taylor CW, McGale P, Kühn T, Poortmans PM, Whelan T, et al. Regional lymph node irradiation in early stage breast cancer: an EBCTCG meta-analysis of 13,000 women in 14 trials. San Antonio Breast Cancer Symp; 2018 Dec 4-8; San Antonio, USA.

4 Corradini S, Krug D, Meattini I, Fastner G, Matuschek C, Cutuli B. Challenges in Radiotherapy. Breast Care (Basel). 2019 Jun;14(3): 152-8.

5 Ditsch N, Untch M, Thill M, Müller V, Janni W, Albert US, et al. AGO Recommendations for the Diagnosis and Treatment of Patients with Early Breast Cancer: Update 2019. Breast Care (Basel). 2019 Aug;14(4):224-45.
6 Thill M, Jackisch C, Janni W, Müller V, Albert US, Bauerfeind I, et al. AGO Recommendations for the Diagnosis and Treatment of $\mathrm{Pa}$ tients with Locally Advanced and Metastatic Breast Cancer: Update 2019. Breast Care (Basel). 2019 Aug;14(4):247-55.

7 Krug D, Baumann R, Budach W, Duma M. N, Dunst J, Feyer P, Fietkau R, Haase W, Harms W, Hehr T, Piroth M, D, Sedlmayer F, Souchon R, Wenz F, Sauer R: Commercially available gene expression assays as predictive tools for adjuvant radiotherapy? A critical review. Breast Care (Basel). 2020;15(2). doi: 10.1159/000505656.

8 Forster T, Köhler C. V, K, Debus J, HörnerRieber J: Accelerated partial breast irradiation: a new standard of care? Breast Care (Basel). 2020;15(2). doi: 10.1159/000506254.

9 Poortmans PM, Collette S, Kirkove C, Van Limbergen E, Budach V, Struikmans H, et al.; EORTC Radiation Oncology and Breast Cancer Groups. Internal Mammary and Medial Supraclavicular Irradiation in Breast Cancer. N Engl J Med. 2015 Jul;373(4):317-27.

10 Thorsen LB, Offersen BV, Danø H, Berg M, Jensen I, Pedersen AN, et al. DBCG-IMN: A Population-Based Cohort Study on the Effect of Internal Mammary Node Irradiation in Early Node-Positive Breast Cancer. J Clin Oncol. 2016 Feb;34(4):314-20.
11 Whelan TJ, Olivotto IA, Parulekar WR, Ackerman I, Chua BH, Nabid A, et al.; MA.20 Study Investigators. Regional Nodal Irradiation in Early-Stage Breast Cancer. N Engl J Med. 2015 Jul;373(4):307-16.

12 Borm KJ, Kessel K, Devecka M, Muench S, Straube C, Schiller K, et al. Variability in lymph node irradiation in patients with breast cancer-results from a multi-center survey in German-speaking countries. Strahlenther Onkol. 2020 Jan;196(1):15-22.

13 Duma MN, Baumann R, Budach W, Dunst J, Feyer P, Fietkau R, et al.; Breast Cancer Expert Panel of the German Society of Radiation Oncology (DEGRO). Heart-sparing radiotherapy techniques in breast cancer patients: a recommendation of the breast cancer expert panel of the German society of radiation oncology (DEGRO). Strahlenther Onkol. 2019 Oct;195(10):861-71.

14 Duma MN. An update on regional nodal irradiation: indication, target volume delineation and radiotherapy techniques. Breast Care (Basel). 2020;15(2). doi: 10.119/000507040.

15 Matuschek C, Nestle-Kraemling C, Kühn T, Fehm T, Boelke E, Corradini S, et al. Maas K, Seidel C, Budach W. Neoadjuvant radio(chemo)therapy for breast cancer: an old concept Revisited. Breast Care (Basel). 2020; 15(2). doi: 10.1159/000507041.

16 Corradini S, Krug D, Meattini I, Matuschek C, Bölke E, Francolini G, et al. Preoperative radiotherapy: a paradigm shift in the treatment of breast cancer? A review of literature. Crit Rev Oncol Hematol. 2019 Sep;141:102-11. 